\title{
Modeling Soot Oxidation with the Extended Quadrature Method of Moments
}

\author{
Achim Wick ${ }^{\mathrm{a}, *}$, Tan-Trung Nguyen ${ }^{\mathrm{b}, \mathrm{c}}$, Frédérique Laurent ${ }^{\mathrm{b}, \mathrm{c}}$, \\ Rodney O. Fox ${ }^{\mathrm{b}, \mathrm{c}, \mathrm{d}}$, Heinz Pitsch ${ }^{\mathrm{a}}$ \\ ${ }^{a}$ Institute for Combustion Technology, RWTH Aachen University, 52062 Aachen, \\ Germany \\ ${ }^{b}$ Laboratoire EM2C, CNRS, CentraleSupélec, Université Paris-Saclay, Grande Voie des \\ Vignes, 92295 Châtenay-Malabry cedex, France \\ ${ }^{c}$ Fédération de Mathématiques de l'Ecole Centrale Paris, FR CNRS 3487, France \\ ${ }^{d}$ Department of Chemical and Biological Engineering, 2114 Sweeney Hall, Iowa State \\ University, Ames, IA 50011-2230, USA
}

\begin{abstract}
Modeling the oxidation of soot particles in flames is a challenging topic both from a chemical point of view and regarding the statistical treatment of the evolution of the soot Number Density Function (NDF). The Method of Moments is widely-used for the statistical modeling of aerosol dynamics in various applications, and a number of different moment methods have been established and successfully applied to the modeling of soot formation and growth. However, a shortcoming of existing moment methods is the lack of an accurate, numerically robust, and computationally efficient way to treat soot oxidation, especially regarding the prediction of the particle number density. In this work, the recently developed Extended Quadrature Method of Moments (EQMOM) is integrated with a physico-chemical soot model and combined with a treatment for particle removal by oxidation. This leads to
\end{abstract}

\footnotetext{
*Corresponding author

Email address: a.wick@itv.rwth-aachen.de (Achim Wick) 
a modeling framework for the simulation of coupled inception, growth, coagulation, and oxidation of soot in flames. In EQMOM, the moment equations are closed by reconstructing the soot NDF with a superposition of continuous kernel functions. Various standard distribution functions can be used as kernel functions, and the algorithm has been implemented here using gamma and lognormal distributions. It is shown that and discussed why gamma distributions are more suitable as kernel functions than lognormal distributions in order to accurately predict soot oxidation. The integrated model is validated by comparisons with analytical solutions for the NDF, results from Monte Carlo simulations of soot formation and oxidation in flames, and experimental data.

Keywords: Soot oxidation, method of moments, EQMOM, statistical soot model

\section{Introduction}

In the development of engineering devices, such as next-generation aero or diesel engines, one of the main concerns is the reduction of particulate emissions. While reactive Computational Fluid Dynamics are already widely-used in the design process, a substantial barrier for an extended use of numerical simulations with respect to emissions reduction is a lack in the predictive quality of models for soot formation, and especially for soot oxidation. Aero engines designed following the rich-burn/quick-quench/lean-burn concept are an example for applications in which soot oxidation is very important. While a lot of soot is formed in the primary rich combustion zone, more than $95 \%$ of the particles are oxidized before reaching the exit of the combustor. There- 
fore, in order to accurately predict the amount of soot emissions, reliable models for soot oxidation are crucial.

In addition to the gas-phase chemistry, soot models consist of two main building blocks: First, a physico-chemical model, which describes the various micro-processes of soot inception, growth, and oxidation on a single-particle level. Second, as not every single particle can be tracked in a simulation, a statistical model is required. Statistical methods applicable to soot modeling include Monte Carlo methods, sectional methods, and moment methods. The goal of the present paper is the integration of a recently developed advanced quadrature-based moment method [1] with soot models for an improved statistical description of soot evolution, especially of soot oxidation.

Targets for the development of statistical soot models are: high accuracy, computational efficiency, numerical robustness (related to moment realizability), applicability to 3D turbulent simulations (i.e. relevant for engineering applications), easy implementation, and applicability to multivariate models, which parameterize the particles with more than one quantity. While Monte Carlo (MC) methods $[2,3]$ are very accurate and well suitable for multivariate models, they are computationally expensive, and their applicability is restricted to simplified configurations. However, due to their negligible error, they can serve as a reference solution, which other methods can be validated against.

The most widely-used class of methods is the Method of Moments including the Method of Moments with Interpolative Closure (MOMIC) [4], the (Direct) Quadrature Method of Moments (QMOM/DQMOM) [5, 6], and the Hybrid Method of Moments (HMOM) [7]. The quadrature-based moment 
methods have been shown to be very accurate for the description of aerosol dynamics including particle growth and coagulation. While DQMOM is computationally efficient and can easily be extended to multivariate models, it can become numerically unstable. QMOM, which is numerically robust, has recently been extended to the Conditional QMOM [8], which combines accuracy, robustness, and efficiency, and has also been applied in 2D simulations [9]. However, all of the mentioned methods have difficulties in dealing with soot oxidation in a mathematically rigorous manner. Often, relatively crude assumptions must be made due to shortcomings in the statistical methods [10]. These shortcomings are discussed in more detail in the next section.

Recently, the Extended Quadrature Method of Moments (EQMOM) has been introduced $[1,11]$ as a statistical approach to solve the Population Balance Equation (PBE). This method retains the accuracy and efficiency of the QMOM algorithm, and has already been applied to soot formation and growth processes [12]. However, although, for reasons discussed below, EQMOM is especially suitable to accurately describe soot oxidation and the related disappearance of particles, a formulation for soot oxidation has not yet been investigated.

Soot oxidation is also challenging from a chemical point of view, and it is an active field of research, both experimentally [13] and numerically [14]. It should be noted that the goal of the present paper is not the improvement of chemical soot models. However, in order to benefit from advances of the chemical description of soot oxidation in simulations of engineering interest, high-fidelity statistical methods applicable to soot oxidation must be developed, and the purpose of this paper is to contribute to this development. 
The following section provides a more detailed analysis why the statistical description of soot oxidation is challenging within the framework of moment methods. In section 3, two variants of EQMOM are discussed. Section 4 briefly describes the physico-chemical soot model. Then, the performance of EQMOM is analyzed in the context of two validation cases: First, pure soot oxidation is studied in section 5.1. Second, in section 5.2, the model is validated for a laminar premixed flame with coupled soot growth and oxidation.

\section{Why Soot Oxidation is a Challenge for Moment Methods}

The statistics of a soot particle population are embodied in the Number Density Function (NDF). For a spatially homogeneous system, the governing PBE for the NDF, $n(t, V)$, based on particle volume, $V$, and dependent on time, $t$, reads

$$
\frac{\partial n(t, V)}{\partial t}+\sum_{i} \frac{\partial}{\partial V}\left[g_{i}(t, V) n(t, V)\right]=\sum_{j} \dot{S}_{j} .
$$

The summation over $j$ on the r.h.s. includes the discontinuous source terms of

nucleation, $\dot{S}_{\text {nucl }}$, and coagulation, $\dot{S}_{\text {coag }}$, which can be expressed as integrals of the NDF. The summation over $i$ on the l.h.s. includes all continuous source terms, i.e. surface reactions leading to particle growth and oxidation. The growth rates of these processes, $g_{i}$, appear as convective velocities in phase space.

The volume-moments of the NDF are defined as

$$
m_{k}(t)=\int_{0}^{\infty} V^{k} n(t, V) \mathrm{d} V
$$


where $k$ is the order of the moment. Transport equations for the moments can be derived by multiplication of Eq. 1 with $V^{k}$ and subsequent integration over the phase space:

$$
\begin{aligned}
\frac{\mathrm{d} m_{k}}{\mathrm{~d} t} & =-\left.\sum_{i} V^{k} g_{i}(t, V) n(t, V)\right|_{0} ^{\infty} \\
& +\sum_{i} \int_{0}^{\infty} k V^{k-1} g_{i}(t, V) n(t, V) \mathrm{d} V \\
& +\sum_{j} \int_{0}^{\infty} V^{k} \dot{S}_{j} \mathrm{~d} V .
\end{aligned}
$$

On the r.h.s. of Eq. 3, there are integral terms as well as boundary terms resulting from the integration by parts of the hyperbolic growth terms (second term in Eq. 1). As the support interval of the NDF is semi-infinite, only the boundary term at the minimum particle size (zero in this case) is of concern. If $g_{i}$ is positive, as in the case of surface growth, the term is zero, but if $g_{i}$ is negative, as in the case of oxidation, the boundary flux is non-zero and needs to be computed. Hence, as long as oxidation is excluded, the r.h.s. of Eq. 3 contains only integral terms. In presence of oxidation, the additional flux term appears.

The Method of Moments seeks the closure of the generally unclosed moment source terms, which enables the solution of Eq. 3. The time evolution of the moments represents the evolution of the statistics of the soot NDF. While the zeroth and first order moments are related to the soot number density and volume fraction, respectively, typically a few more moments are solved for in order to obtain a more accurate evaluation of the moment source terms. The evaluation of the boundary flux is especially challenging as it requires the knowledge of the pointwise values of the NDF, which are not directly 
available from the moments.

For the following discussion, it is important to distinguish between the terms "representation of the NDF" and "approximation of the NDF". In classical quadrature-based moment methods, such as (D)QMOM, from the computed set of moments, a multi-delta function is reconstructed, which constitutes a representation of the NDF. The goal of the representation of the NDF is to accurately reproduce its statistics in terms of moments with the assumption that this will help to accurately predict the source terms in the moment equations, while the shape of the reconstructed NDF might be completely different from the true NDF. As long as oxidation is not considered, all source terms appear as integrals of the NDF, and using the (D)QMOM representation for their evaluation is equivalent to a Gaussian quadrature. This method has been shown to be very accurate for soot growth [15].

In case of oxidation, the additional boundary term cannot be closed with such a quadrature, because the pointwise flux at the minimum particle size needs to be known. Therefore, an approximation of the NDF is required, i.e. a reconstruction of the NDF which not only reproduces its statistics, but also accurately approximates its shape, especially close to the minimum particle size. Only from the knowledge of the number density of the smallest particles that will be oxidized during the next time step, the disappearance rate of particles can be computed. This quantity is not available in (D)QMOM, and modeling assumptions are required to compute the decrease in the number density due to oxidation.

In the original variant of MOMIC [4], a transport equation for the moment of order minus infinity is solved, and in HMOM [7], a delta function at the 
size of nucleating particles is introduced. Although the main purpose of adding these equations was not the treatment of oxidation, they can be used to determine the number of the smallest particles. However, the issue is shifted to the question of how many particles are transferred from larger size classes into the first bin. The pointwise knowledge of the NDF would be required to answer this question. However, this information is not available in interpolation-based or standard quadrature methods, and model assumptions need to be introduced.

In order to close this gap, EQMOM has recently been developed. Its moment inversion algorithm provides a continuous function as approximation of the NDF, which is used to evaluate the source terms and the oxidation boundary flux.

\section{EQMOM for Soot Formation and Oxidation}

\subsection{Moment Inversion Algorithm}

The concept of EQMOM relies on the replacement of the delta-functions in (D)QMOM with continuous kernel functions. The NDF is hence approximated by a superposition of $N$ continuous kernel functions, $\delta_{\sigma}\left(V ; V_{\alpha}\right)$, which are weighted by $w_{\alpha}$ :

$$
n(V) \approx \sum_{\alpha=1}^{N} w_{\alpha} \delta_{\sigma}\left(V ; V_{\alpha}\right)
$$

The moment inversion then consists in reconstructing such kind of NDF from its moments of order 0 to $2 N$, i.e., finding the non-negative weights, $w_{\alpha}$, the abscissas of the kernel functions, $V_{\alpha}$, and their shape parameter, $\sigma$, which is identical for all kernel functions. Once these parameters are determined, 
the moment source terms in integral form can be computed with a so-called secondary quadrature, that is a quadrature for each kernel function [1]. The boundary term in Eq. 3 can be computed from the approximated NDF, as described in Sec. 3.4.

Different kinds of kernel functions are possible, which all satisfy the two following constraints: First, they formally tend to a Dirac delta function when $\sigma$ tends to zero, thus recovering a quadrature. Second, there is a linear dependence between any moment vector, $\mathbf{m}$, of a reconstructed NDF and the vector $\mathbf{m}^{\star}$ of components $m_{k}^{\star}=\sum_{\alpha=1}^{N} w_{\alpha} V_{\alpha}^{k}$ :

$$
\mathbf{m}=\mathbf{A}(\sigma) \mathbf{m}^{\star} .
$$

The form of $\mathbf{A}(\sigma)$ depends on the specific choice of kernel functions. This second constraint allows the use of the efficient and robust standard quadrature algorithm. Indeed, for any value of $\sigma$, one can compute $\mathbf{m}^{\star}(\sigma)=\mathbf{A}(\sigma)^{-1} \mathbf{m}$ and then the quadrature points $\left(w_{\alpha}(\sigma), V_{\alpha}(\sigma)\right)_{\alpha=1}^{N}$, thus leading to a reconstruction. The moments of order 0 to $2 N-1$ of this reconstruction are thus the given $m_{k}$ and the value of $\sigma$ has then to be adapted in order for its $2 N$ th order moment $\bar{m}_{2 N}(\sigma)$ to be $m_{2 N}$. The algorithm for moment inversion is illustrated in Fig. 1.

A new robust and efficient algorithm for the reconstruction provided in [16], improving the one of [1], is applied here. It uses an efficient way to solve the nonlinear problem $J(\sigma)=0$, where $J(\sigma)=m_{2 N}-\bar{m}_{2 N}(\sigma)$. In some cases, a solution does not exist; then, $J(\sigma)^{2}$ and hence the error on the last moment is minimized. Moreover, the algorithm is able to deal efficiently with moment vectors at the boundary of the space of realizable moments, where the only possible corresponding NDF is a single or multi-delta function. Such 


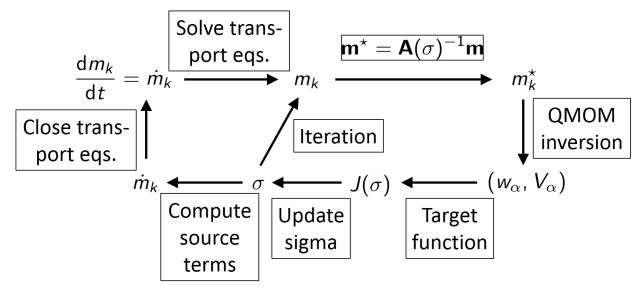

Figure 1: Schematic of the EQMOM algorithm.

cases frequently appear in regions of a flame where soot nucleation has just started. A method then detects if the moment vector is close to the boundary of moment space. If this is the case, the algorithm adaptively switches to the standard quadrature algorithm, until coagulation has broadened the NDF.

Several types of kernel functions can be used, e.g. Gaussian distributions for internal coordinates with an unbounded support interval, or beta distributions if the support interval is bounded. As the support interval for soot particle volume is semi-infinite, $[0, \infty)$, two possible choices for the kernel functions are gamma [1] or lognormal [11] distributions. Both variants are applied and compared in this paper.

\subsection{Gamma EQMOM}

The kernel functions for gamma EQMOM [1] are given as

$$
\delta_{\sigma}\left(V ; V_{\alpha}\right)=\frac{V^{\lambda_{\alpha}-1} \mathrm{e}^{-V / \sigma}}{\Gamma\left(\lambda_{\alpha}\right) \sigma^{\lambda_{\alpha}}},
$$

where $\lambda_{\alpha}=V_{\alpha} / \sigma$, and $\Gamma$ is the Gamma function. The corresponding moments can be analytically expressed as

$$
m_{0}=\sum_{\alpha=1}^{N} w_{\alpha}, \quad m_{k}=\sum_{\alpha=1}^{N} w_{\alpha} \prod_{i=0}^{k-1}\left(V_{\alpha}+i \sigma\right), \quad k \geq 1 .
$$


The matrix $\mathbf{A}(\sigma)$ is then triangular with coefficients $[\mathbf{A}(\sigma)]_{i, j}=a_{i, j} \sigma^{i-j}$ for $0 \leq j \leq i$, where $a_{k, k}=1$ and $a_{k, i}$ is given by the following recurrence formula [16]:

$$
a_{k, i}=(k-1) a_{k-1, i}+a_{k-1, i-1}, \quad k \geq 1, \quad i=2, \ldots, k-1 .
$$

\subsection{Lognormal EQMOM}

The lognormal kernel functions are

$$
\delta_{\sigma}\left(V ; V_{\alpha}\right)=\frac{1}{V \sigma \sqrt{2 \pi}} \exp \left(\frac{-\left(\ln (V)-\ln \left(V_{\alpha}\right)\right)^{2}}{2 \sigma^{2}}\right) .
$$

The moments take the form

$$
m_{k}=\sum_{\alpha=1}^{N} w_{\alpha} V_{\alpha}^{k} \exp \left(\frac{1}{2} k^{2} \sigma^{2}\right)
$$

leading to the matrix $\mathbf{A}(\sigma)$ being diagonal with diagonal coefficients $\exp \left(\frac{1}{2} k^{2} \sigma^{2}\right)$. Moreover, the nonlinear problem is then solved for $\exp \left(\sigma^{2} / 2\right)$ instead of $\sigma$ [11].

\subsection{Treatment of Oxidation}

Operator splitting is applied for the time integration of the moments. The soot growth and coagulation terms are integrated first. Then, oxidation is accounted for using an adaptation to an odd number of moments [16] of the scheme by Massot et al. [17]. The NDF is first reconstructed according to the EQMOM algorithm. Then, eliminating the disappearance flux, i.e. the part of the reconstructed distribution, which is oxidized during the current time step, the corresponding partial moments, are computed analytically (gray region in Fig. 2):

$$
\widetilde{m}_{k}(t)=\int_{V_{\text {Ox }}}^{\infty} V^{k} n(t, V) \mathrm{d} V, \quad k=0,1, \ldots, 2 N+1 .
$$



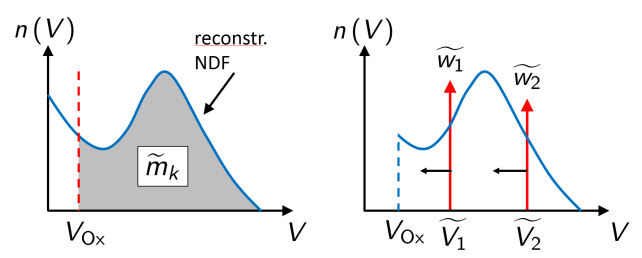

Figure 2: Schematic illustration of the oxidation treatment.

The size of the largest particle that is oxidized in the time step, $V_{\mathrm{Ox}}$, can be evaluated from the oxidation rate.

An $(N+1)$-point quadrature, $\left(\widetilde{w}_{i}, \widetilde{V}_{i}\right)$, corresponding to these modified moments, $\left\{\widetilde{m}_{k}\right\}_{k=0}^{2 N+1}$, is computed. The abscissas are then convected in phase space according to the oxidation rate, and the updated moments are computed as

$$
m_{k}(t+\Delta t)=\sum_{i=1}^{N+1} \widetilde{w}_{i} \widetilde{V}_{i}^{k}(t+\Delta t)
$$

A very important property of this algorithm is that it ensures the realizability of the moment set $[17,16]$.

\section{Soot Model}

For the flames discussed in the following, detailed chemistry computations of the gas phase were performed using the FlameMaster code [18] and the chemical kinetic mechanism of Narayanaswamy et al. [19], which was developed with special focus on soot precursors, and which contains PAH (polycyclic aromatic hydrocarbons) chemistry up to four-ringed molecules. Two-way coupling between gas phase and particulate phase is applied to account for the removal of PAH from the gas phase during soot inception and growth. 
The following soot processes are included in the computations of the particle phase: nucleation using the dimerization model by [15], condensation of PAH dimers, coagulation in the transition regime, surface growth modeled with the HACA mechanism [20,21], with reactions rates given by [15], and oxidation by $\mathrm{OH}$ and $\mathrm{O}_{2}$, with rates given by [15] and references therein. Thermophoretic effects are also included.

\section{Results}

For a thorough validation, model-predicted results are compared to Monte Carlo simulations as well as experimental data from three different experiments. First, we will consider two analytical cases for pure oxidation starting from realistic initial size distributions experimentally determined in [13, 22]. Then, the model will be further validated with an experimental data set of laminar premixed flames [23], focusing on oxidation.

\subsection{Results for Pure Oxidation}

First, soot oxidation is considered isolated from other processes. The goal of this validation case is to test if the EQMOM reconstruction yields a good approximation of realistic soot NDFs at all times during the oxidation process, which is necessary for the accurate prediction of the moments, especially of $m_{0}$ representing the number density.

The burner-stabilized stagnation (BSS) flame approach has been established as an experimental technique to measure the soot NDF in laminar premixed flames [22, 24]. Experiments in this configuration have shown that at downstream locations, bimodal shapes are observed for the soot NDFs in 
a rich $\mathrm{C}_{2} \mathrm{H}_{4} / \mathrm{O}_{2} / \mathrm{Ar}$ flame [22]. In a similar flame that was recently experimentally studied by the same research group [13], a unimodal NDF in the flame has been found. Both cases are interesting test cases, as both NDFs might be present at some point in a turbulent flame and be subjected to strong oxidation when reaching the flame front. Therefore, both cases will be considered here and used as initial conditions for the oxidation test cases described in the following.

\subsubsection{Analytical Solution}

Both gamma and lognormal EQMOM are applied using both experimentally measured soot NDFs discussed above (flame $\mathrm{C} 3$ in $[22]$ at $\mathrm{HAB}=8 \mathrm{~mm}$ and $\mathrm{C}_{2} \mathrm{H}_{4}$ flame in [13]) as initial conditions. For pure oxidation, if the initial $\mathrm{NDF}$ is given, the temporal evolution of the NDF and its moments can be obtained analytically from the oxidation law. As the goal is the validation of the statistical part of the model, a relatively simple chemical model is applied, and results are compared to the analytical solution. The oxidation rate is taken proportional to the particle surface, and particles are assumed to be spherical. The particle diameter is taken as internal coordinate of the NDF, such that oxidation simply shifts the initial NDF to smaller sizes, and the evolution of the NDF and its moments can easily be computed. As particles smaller than $2.5 \mathrm{~nm}$ are below the detection limit of the particle sampler, a small part of the initial NDF is unknown. Therefore, the comparison of EQMOM and the analytical solution starts at the time when the smallest measured particle has reached zero size. These simplifications do not lead to a loss of generality regarding the conclusions drawn for the performance of the statistical model. 


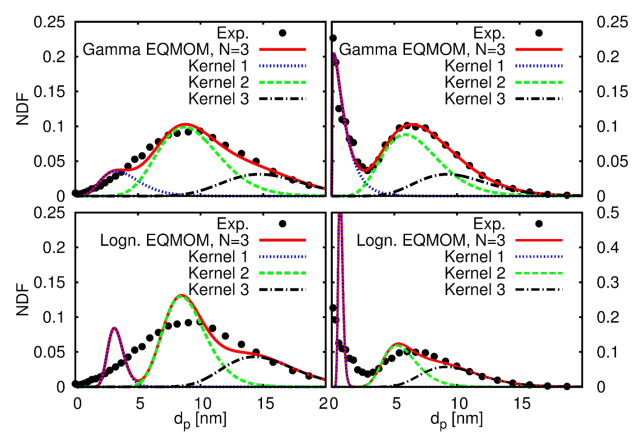

Figure 3: Reconstruction of two experimentally measured, normalized NDFs from rich, premixed ethylene flames (left: [13]; right: [22]) using gamma EQMOM (upper row) and lognormal EQMOM (lower row) with three kernel functions.

5.1.2. Discussion: Gamma EQMOM More Suitable than Lognormal EQ$M O M$

From the experimental NDFs, the diameter-moments are computed as initial conditions, and the EQMOM reconstruction for this first validation case is based on diameter-moments. During the oxidation process, the NDF is reconstructed in every time step using the EQMOM algorithm of Sec. 3.1. Then, the oxidation algorithm of Sec. 3.4 is applied. Figure 3 shows the reconstructed initial NDFs using gamma and lognormal EQMOM with three kernel functions, i.e. seven moments need to be transported. The reconstructions of the NDFs at different times during the simulation are provided in the Supplementary Material.

Both the unimodal and the bimodal NDF can be very well approximated using two (not shown here) or three gamma distributions, while lognormal EQMOM is less accurate. The lognormal kernels do not overlap very much, which leads to a bimodal shape for both NDFs; also the experimentally 

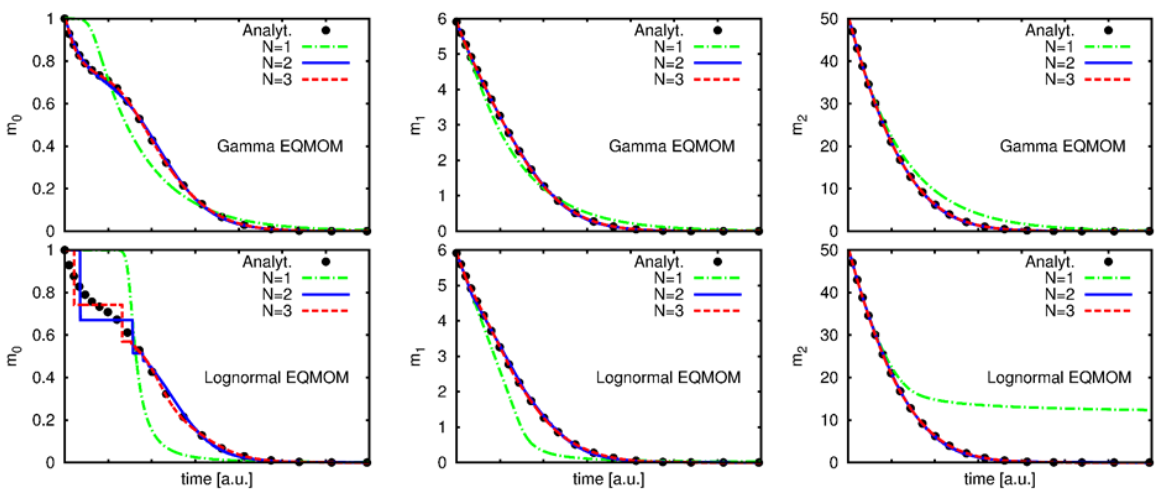

Figure 4: Time evolution of the first three moments, representing number density, soot volume fraction, and variance of the NDF, normalized with the initial particle number, during pure oxidation of the soot population represented by the bimodal NDF in Fig. 3 .

bimodal NDF is not well approximated in the region of small particles. Lognormal EQMOM has difficulties to capture non-zero values at the minimum particle size, because the lognormal distribution always starts at zero. In gamma EQMOM, depending on the parameters of the gamma distributions, a smooth transition occurs between the NDF starting at zero and at a nonzero value. Although the parameter $\sigma$ is identical for all kernels, the shapes of the kernels can differ in that the first kernel starts at a non-zero value, while the others start at zero. This is a results of the definition of $\sigma$ and the parameter of the gamma distribution, $\lambda_{\alpha}=V_{\alpha} / \sigma$, and it enables an accurate approximation of the NDF, especially for small particle sizes, which is important for an accurate prediction of the number density during oxidation.

Figure 4 shows the time evolution of $m_{0}, m_{1}$, and $m_{2}$, representing number density, soot volume fraction, and variance of the NDF, respectively, during 
oxidation of the bimodal NDF. Oxidation of this NDF is the more general case and is therefore discussed here; corresponding results for the unimodal NDF are provided in the Supplementary Material. EQMOM with just one kernel function, i.e. three transported moments, does not yield sufficient accuracy. Using two or more kernels, i.e. five or more moments, both gamma and lognormal EQMOM can very accurately predict the soot volume fraction and higher moments. Gamma EQMOM also excellently predicts the evolution of the number density. Due to the bimodality of the NDF, it first decreases fast until the peak of small particles has been oxidized, then the rate slows down, before increasing again when the second peak is oxidized. This behavior is qualitatively and quantitatively very well captured.

For modeling the disappearance rate of particles during oxidation using traditional moment methods, it is often assumed that a particle is removed after the mass of an average-sized particle has been oxidized. This model assumption couples the rate of change of number density to the rate of change of volume fraction. This coupling is obviously wrong, especially for the typical bimodal NDFs, and can only be avoided if the pointwise values of the NDF are known with sufficient accuracy, as is the case in gamma EQMOM.

Lognormal EQMOM produces jumps in the number density. This behavior is linked to the evolution of the parameters of the kernel functions. Figure 5 shows the weights and abscissas, and Fig. 6 shows the evolution of the shape parameter, $\sigma$. While the weights of the gamma distributions follow smooth curves, the weights of the lognormal distributions show jumps, whose positions are dependent on the time step size (not shown here). During oxidation, the first lognormal kernel becomes very narrow, until it disappears 


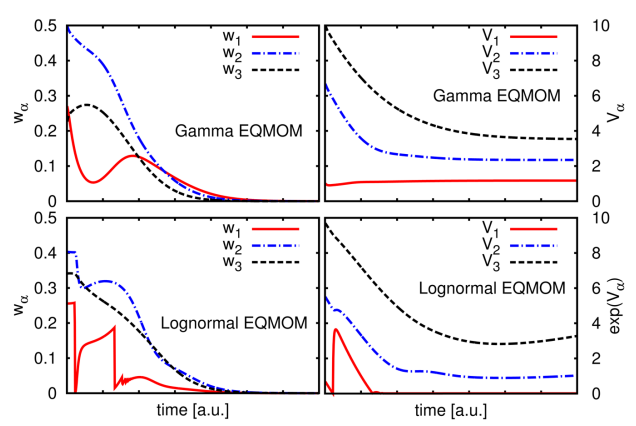

Figure 5: Time evolution of the weights and abscissas of the kernel functions during pure oxidation of the soot population represented by the bimodal NDF in Fig. 3.

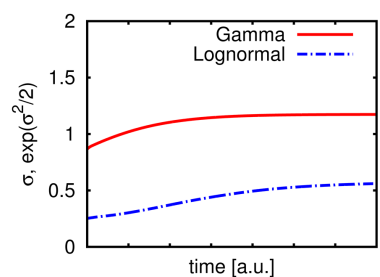

Figure 6: Time evolution of the shape parameter $\sigma$. Same case as in Fig. 5. 


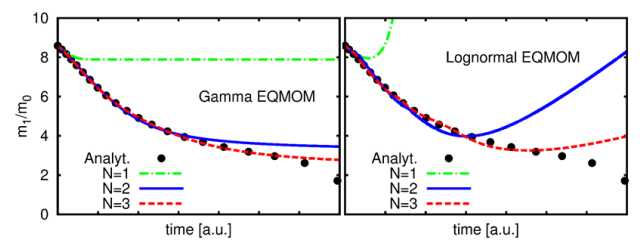

Figure 7: Time evolution of $m_{1} / m_{0}$. Same case as in Fig. 4.

in a certain time step, which leads to the jump in the number density. The gamma distributions are always broad, and, as discussed above, the kernels have different parameters $\lambda_{\alpha}=V_{\alpha} / \sigma$ for a common value of $\sigma$, such that the first kernel starts at a non-zero value, while the other kernels start at zero. This behavior helps to avoid the sudden disappearance of a kernel and enables an accurate prediction of the number density, which is also independent of the time step size.

Another benefit of gamma EQMOM over lognormal EQMOM is evident from Fig. 7, which shows the time evolution of $m_{1} / m_{0}$, corresponding to a mean particle diameter. Gamma EQMOM is able to accurately predict the mean particle diameter even for large times, when both $m_{1}$ and $m_{0}$ go to zero. For lognormal EQMOM, in contrast, the convergence with increasing number of transported moments is much slower, and for large times, the monotonically decreasing behavior is not captured any more.

\subsection{Results for Coupled Soot Formation and Oxidation}

The two-stage burner experiment by Neoh et al. [23] is simulated to validate the EQMOM algorithm for combined soot formation, growth, coagulation, and oxidation. In this experiment, soot is produced in a rich, premixed, burner-stabilized $\mathrm{CH}_{4}$ /air flame. Then, secondary air is added, and the soot 
is oxidized in a second premixed flame (case with $\phi_{\text {global }}=1.15$ in [23] is studied here). Although soot formation and oxidation are dominant in the primary and secondary burner, respectively, all soot processes are relevant and considered in both flames. As the flames are laminar and one-dimensional, the spatial coordinate is transformed into a pseudo-time or Lagrangian time using the particle velocity, i.e. the sum of gas and thermophoretic velocities. The particulate phase is then integrated in time. The simulation results of the primary burner are used as initial conditions for the secondary burner.

\subsubsection{Monte Carlo Simulations}

As an analytical solution for the NDF is no longer possible for this case, EQMOM predictions are here compared to Monte Carlo simulations using the same physico-chemical soot model. In addition, model results are compared to experimental data for the secondary burner.

MC simulations are particularly useful for validation of statistical models, since they require no closure assumption and hence describe the exact NDF evolution for a given physico-chemical model. The MC code uses the algorithm by $[3,25]$ for coagulation in the transition regime. To improve computational efficiency of the MC simulations, the method of majorant kernels $[26]$ is used for the continuum regime.

\subsubsection{Validation: Soot Formation and Oxidation in a Two-Stage Burner}

In Fig. 8, the soot volume fraction in the primary burner is shown on the left. Very good agreement between EQMOM and MC results is obtained. To highlight the effect of oxidation even in this rich flame $(\phi=2.1)$, additional simulations excluding oxidation are also shown. As for both simulations 

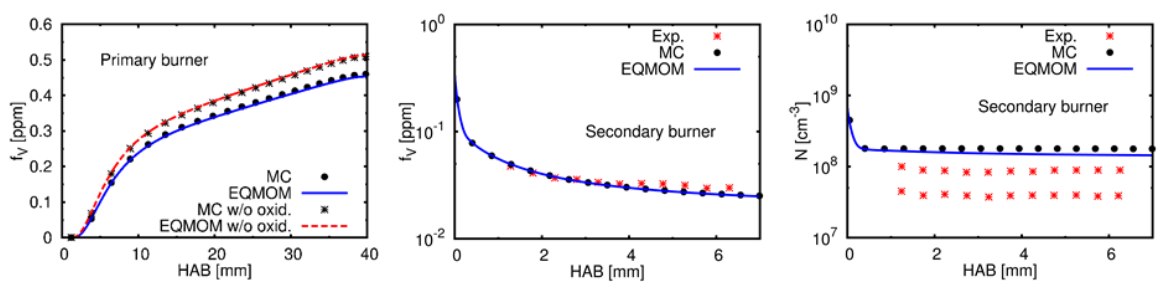

Figure 8: Soot volume fraction in the primary (left) and secondary burner (middle), and number density in the secondary burner (right). Comparison of EQMOM with MC, and with experimental data in the secondary burner from [23], where two postprocessing techniques were used to determine the number density.

the agreement between EQMOM and MC is very good, it can be concluded that EQMOM is suitable to accurately predict isolated soot formation and coagulation as well as simultaneous oxidation.

Figure 8 also shows the evolution of soot volume fraction and number density in the oxidation-dominated secondary burner. Comparisons are made between EQMOM, MC and experimental data. It should be noted that a deviation of EQMOM results from experimental results is always due to a combination of uncertainties in the physico-chemical and the statistical model, while the comparison of EQMOM with MC simulations isolates the statistical error, which is of prime concern here. Regarding the soot volume fraction, EQMOM results are in excellent agreement both with MC simulations and experimental data.

The prediction of the experimental number density is also reasonable, given the experimental uncertainty. The overprediction of the number density might be a result of an underprediction of the coagulation rate in the primary burner, which could be improved with a more sophisticated, multivariate soot model including a model for aggregation. It should also be noted 
that a significant experimental uncertainty can be expected, as Neoh [23] computed the number density using extinction and scattering measurements, and making assumptions about the particle size distribution (monodisperse or single lognormal). More importantly, the EQMOM results for the number density agree well with the MC simulations, which validates the EQMOM approach for coupled soot formation and oxidation.

\section{Conclusions}

The EQMOM algorithm has been integrated with a physico-chemical soot model including soot inception, growth, coagulation, and oxidation processes. Extending existing EQMOM implementations, the algorithm has for the first time been combined with a treatment for particle removal by oxidation. Two variants of EQMOM applicable to NDFs with a semi-infinite support interval, gamma and lognormal EQMOM, have been applied to soot oxidation using realistic soot NDFs taken from two different experiments as initial conditions. While both methods are able to accurately predict the soot volume fraction and higher order moments of the NDF, lognormal EQMOM has difficulties to predict the soot number density. On the contrary, gamma EQMOM using at least two kernel functions yields an excellent approximation of the NDF, and it is therefore a suitable method for the accurate prediction of both soot volume fraction, number density, and the soot NDF itself. A subsequent application of gamma EQMOM to the two-stage burner experiment revealed that this is also true for coupled soot formation and oxidation in flames. 


\section{Acknowledgements}

A.W. and H.P. gratefully acknowledge funding by the German Research Foundation (DFG) under grant no. PI 368/6-1 and by the German Research Association for Combustion Engines e.V. (FVV) under grant no. 1166. T.T.N., F.L. and R.O.F. gratefully acknowledge funding by the French National Research Agency (ANR) under grant ANR-13-TDMO-02 ASMAPE for the ASMAPE project.

\section{References}

[1] C. Yuan, F. Laurent, R. O. Fox, J. Aerosol Sci. 51 (2012) 1-23.

[2] M. Balthasar, M. Kraft, Combust. Flame 133 (2003) 289-298.

[3] R. I. A. Patterson, J. Singh, M. Balthasar, M. Kraft, W. Wagner, Combust. Flame 145 (2006) 638-642.

[4] M. Frenklach, Chem. Eng. Sci. 57 (2002) 2229-2239.

[5] R. McGraw, Aerosol Sci. Tech. 27 (1997) 255-265.

[6] D. L. Marchisio, R. O. Fox, J. Aerosol Sci. 36 (2005) 43-73.

[7] M. E. Mueller, G. Blanquart, H. Pitsch, Combust. Flame 156 (2009) $1143-1155$.

[8] C. Yuan, R. O. Fox, J. Comput. Phys. 230 (2011) 8216-8246.

[9] A. Buffo, M. Vanni, D. L. Marchisio, R. O. Fox, Int. J. Multiphas. Flow 50 (2013) 41-57. 
[10] R. O. Fox, F. Laurent, M. Massot, J. Comput. Phys. 227 (2008) 30583088.

[11] E. Madadi-Kandjani, A. Passalacqua, Chem. Eng. Sci. 131 (2015) 323339.

[12] S. Salenbauch, A. Cuoci, A. Frassoldati, C. Saggese, T. Faravelli, C. Hasse, Combust. Flame 162 (2015) 2529-2543.

[13] J. Camacho, Y. Tao, H. Wang, Proc. Combust. Inst. 35 (2015) 18871894.

[14] M. Sirignano, J. Kent, A. D’Anna, Energ. Fuel. 27 (2013) 2303-2315.

[15] G. Blanquart, H. Pitsch, in: H. Bockhorn, A. D'Anna, A. Sarofim, H. Wang (Eds.), Combustion Generated Fine Carbonaceous Particles, KIT Scientific Publishing, 2009, pp. 439-466.

[16] T. T. Nguyen, F. Laurent, R. O. Fox, M. Massot, Solution of population balance equations in applications with fine particles: mathematical modeling and numerical schemes, 2015. Submitted, available on HAL: https://hal.archives-ouvertes.fr/.

[17] M. Massot, F. Laurent, D. Kah, S. de Chaisemartin, SIAM J. Appl. Math. 70 (2010) 3203-3234.

[18] H. Pitsch, FlameMaster. A C++ computer program for 0D combustion and 1D laminar flame calculations., n.d.

[19] K. Narayanaswamy, P. Pepiot, H. Pitsch, Combust. Flame 162 (2015) $1193-1213$. 
[20] M. Frenklach, H. Wang, Proc. Combust. Inst. 23 (1991) 1559-1566.

[21] M. Frenklach, Phys. Chem. Chem. Phys. 4 (2002) 2028-2037.

[22] A. D. Abid, J. Camacho, D. A. Sheen, H. Wang, Combust. Flame 156 (2009) 1862-1870.

[23] K. G. Neoh, Soot burnout in flames, Ph.D. thesis, Massachusetts Institute of Technology, 1981.

[24] J. Camacho, S. Lieb, H. Wang, Proc. Combust. Inst. 34 (2013) 18531860.

[25] J. Singh, R. Patterson, M. Balthasar, M. Kraft, W. Wagner, Modelling soot particle size distribution: Dynamics of pressure regimes, 2004. Preprint, University of Cambridge.

[26] M. Goodson, M. Kraft, J. Computat. Phys. 183 (2002) 210-232. 
Supplementary Material

Modeling Soot Oxidation with the Extended Quadrature Method of Moments

Achim Wick ${ }^{\star}$, Tan-Trung Nguyen, Frédérique Laurent, Rodney O. Fox, Heinz Pitsch

${ }^{*}$ Corresponding author: a.wick@itv.rwth-aachen.de

\section{Evolution of Moments during Oxidation of the Unimodal NDF}

Fig. 4 in the main paper shows the evolution of the moments during oxidation of the soot population represented by the bimodal NDF. These results are supplemented here by the corresponding results for the unimodal NDF.
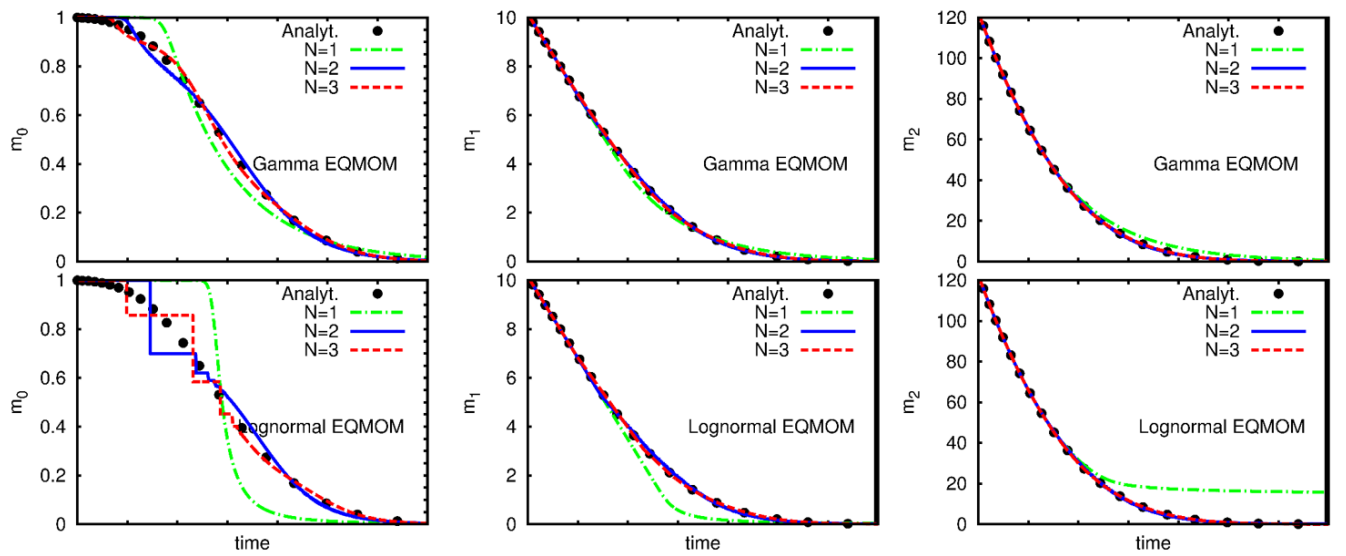

Figure S1: Time evolution of the first three moments, representing number density, soot volume fraction, and skewness of the NDF, normalized with the initial particle number, during pure oxidation of the soot population represented by the unimodal NDF.

\section{Reconstruction of the NDF during Oxidation}

Fig. 3 in the main paper shows the reconstruction of both the unimodal and the bimodal NDF in the first time step using both gamma and lognormal EQMOM with three kernel functions. These results are supplemented here by the reconstructions of the NDF at several times during the oxidation using both gamma and lognormal EQMOM with two and three kernels. 
Supplementary Material

Modeling Soot Oxidation with the Extended Quadrature Method of Moments Achim Wick ${ }^{\star}$, Tan-Trung Nguyen, Frédérique Laurent, Rodney O. Fox, Heinz Pitsch

*Corresponding author: a.wick@itv.rwth-aachen.de

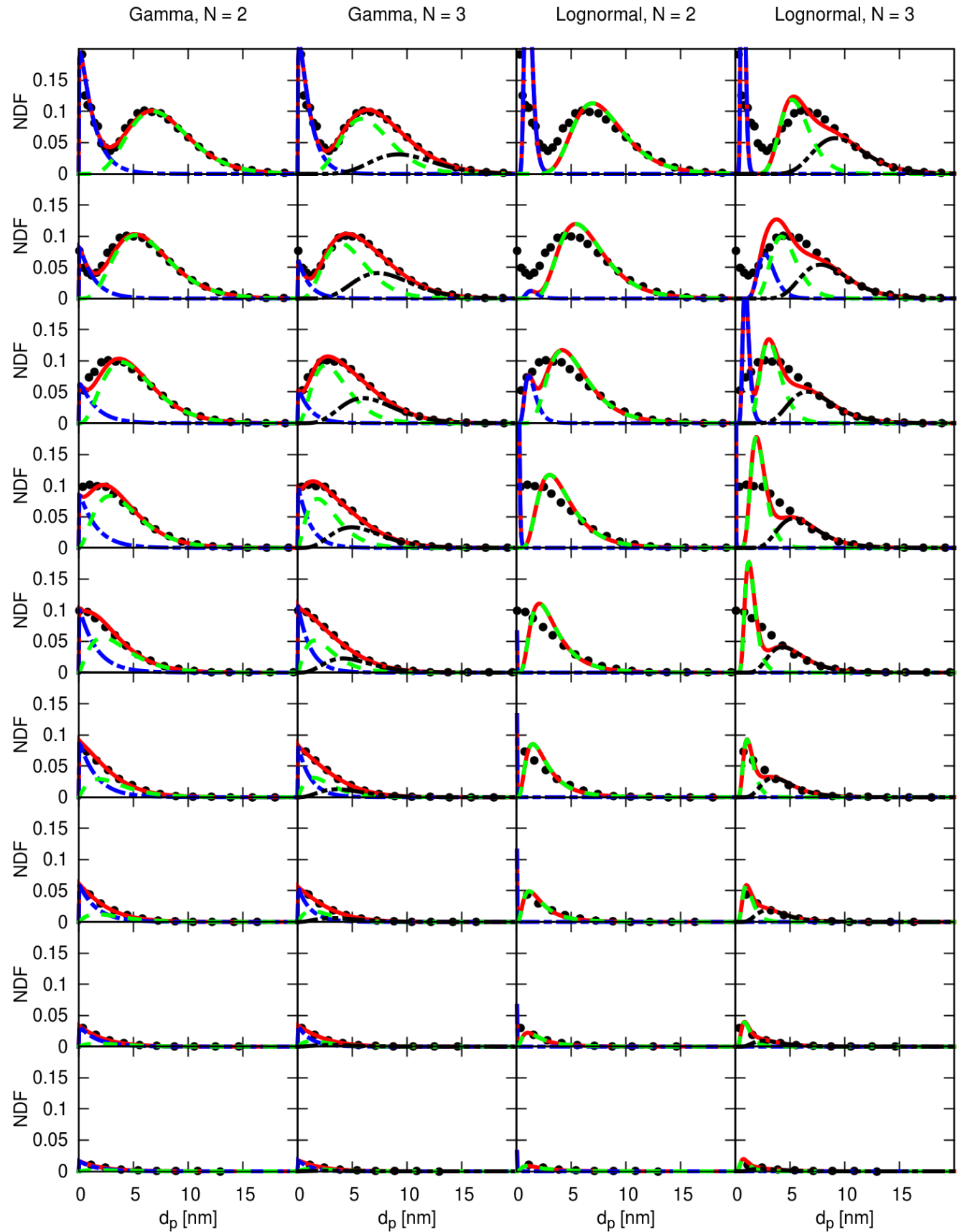

Figure S2: Reconstruction of the NDF at several times during the oxidation of the soot population represented by the bimodal NDF. Gamma and lognormal EQMOM with two and three kernel functions are compared to the initial experimental NDF (top row) and the analytical solution at different times (time increasing from top to bottom). Line types and colors are the same as in Fig. 3 in the main paper. 
Supplementary Material

Modeling Soot Oxidation with the Extended Quadrature Method of Moments Achim Wick ${ }^{\star}$, Tan-Trung Nguyen, Frédérique Laurent, Rodney O. Fox, Heinz Pitsch

*Corresponding author: a.wick@itv.rwth-aachen.de

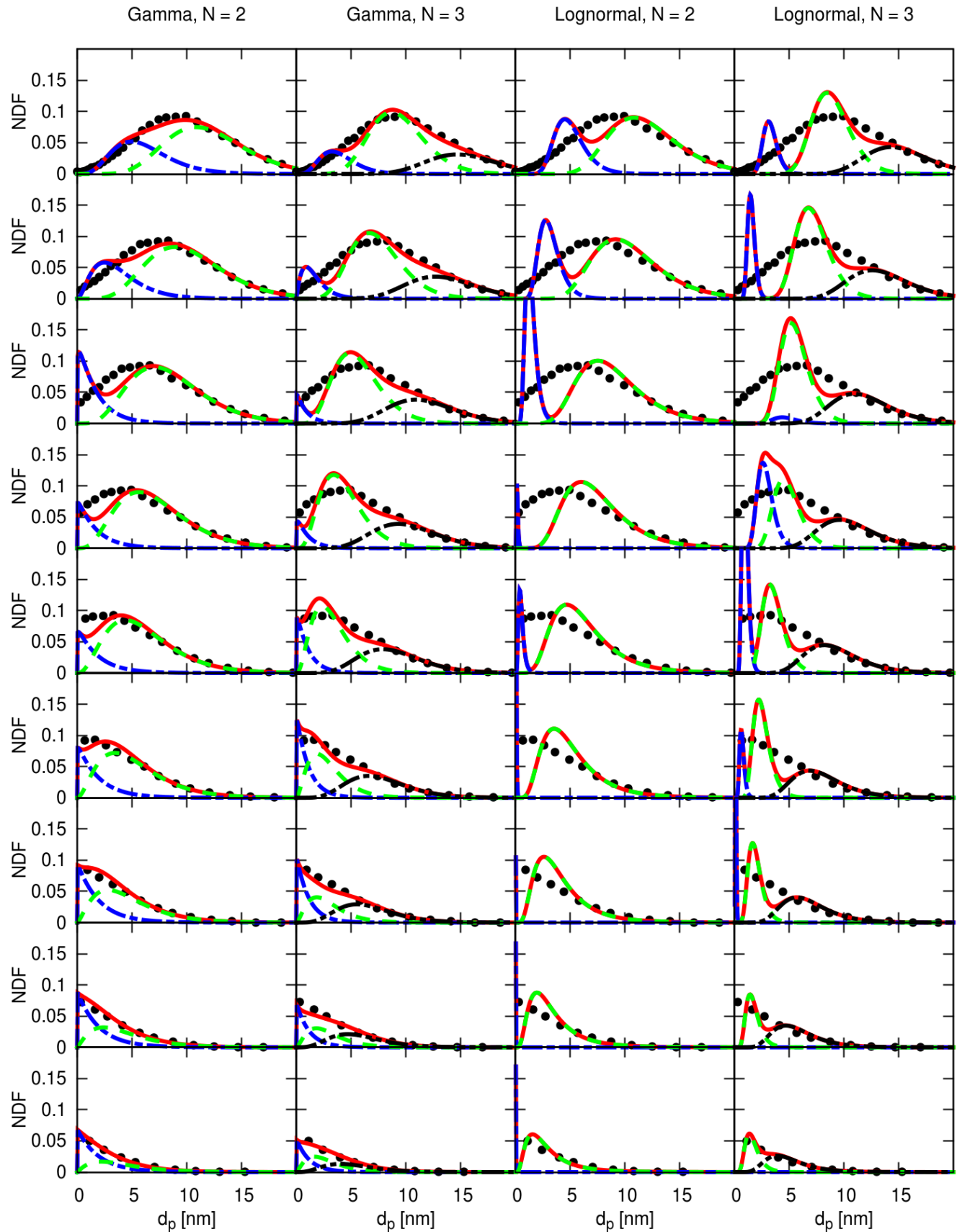

Figure S3: Reconstruction of the NDF at several times during the oxidation of the soot population represented by the unimodal NDF. Gamma and lognormal EQMOM with two and three kernel functions are compared to the initial experimental NDF (top row) and the analytical solution at different times (time increasing from top to bottom). Line types and colors are the same as in Fig. 3 in the main paper. 